\title{
PN Code Acquisition in Multiplicative and Weakly Dependent Non- Gaussian Noises based on Locally Optimum Detection Statistic
}

\author{
Feng Shen and Guanghui $\mathrm{Xu}$ \\ College of Automation, Harbin Engineering University, Harbin, China \\ sf407@126.com
}

\begin{abstract}
In most previously reported studies on pseudonoise $(P N)$ code acquisition, independent additive noises have been assumed in various noise environments. The use of an independent observation model may cause considerable performance degradation in modern high datarate communication systems. In these studies, only additive noise model is considered. In this paper, a new detector for PN code acquisition in multiplicative and weakly dependent nonGaussian noises is proposed. Modeling the acquisition problem as a hypothesis testing problem, the test statistical is derived for multiplicative and weakly dependent non-Gaussian noises, based on the locally optimum detection technique. Numerical results show that the proposed detector can offer substantial performance improvement over the conventional schemes in multiplicative and weakly dependent non-Gaussian noises.
\end{abstract}

Keywords: direct sequence spread spectrum; locally optimum detection; multiplicative noise; weakly dependent non-Gaussian noises; code acquisition

\section{Introduction}

Rapid establishment of code acquisition is an important technical issue in direct-sequence spread-spectrum (DS-SS) systems. The basic unit in an acquisition system is a decisionmaking device or a detector. Most schemes proposed for rapid code acquisition employ the squared-sum (SS) detector with a noncoherent in-phase/quadrature-phase (I-Q) correlator [1]. This is because the SS detector is optimized for Gaussian noise channels, and the statistics due to fading in acquisition systems can usually be modeled as Gaussian processes by virtue of the central limit theorem. Some schemes are proposed for code acquisition systems in applications with non-Gaussian noises [2-3], such as atmospheric and man-made noise arising in indoor/outdoor mobile communication systems. These acquisition schemes are also proposed for the additive noise model, in which noise is added to a signal to generate observations. In some different types of situations, such as the multipath or reverberation phenomena and the actions of automatic gain control circuits or of nonlinearities acting on additive signal and noise components, the multiplicative noise models have to be considered [4-5].

In a number of the studies [4-8], the signal detection in multiplicative noise has been explored. But those researches are limited only in one-dimensional signal detection. And in these studies, independent noises have been assumed in various noise environments, however, is frequently violated, especially in modern discrete-time signal-detection applications, where the noise components contained in the sampled data tent to have dependence due to a very high sampling rate. As a consequence, a detector optimized for independent noise is often not guaranteed to be optimum in practical signal detection systems, which becomes more critical as the sampling rate increases.

In this paper, we focus on the code acquisition in multiplicative and weakly dependent non-Gaussian noise environments. In particular, we consider the locally optimum detection of DS/SS signal based on the generalized version of the Neyman-Pearson fundamental lemma 
[9-11] of statistical hypothesis testing. Locally optimum (LO) detectors are optimum in detecting weak signals, since an LO detector has the maximum slope of its power function when the signal-to-noise ratio (SNR) approaches zero [12-13]. Although much effort has been devoted to the code acquisition using LO detectors in non-Gaussian or Gaussian noise environments [12-14], only the independent and additive noise model has been considered. In the rest of this paper, we will employ locally optimum (LO) detectors for code acquisition systems in multiplicative and weakly dependent non-Gaussian noises.

The remainder of this paper is organized as follows. In section 2 the observation model of the received signal and the hypothesis testing model in multiplicative and weakly dependent non-Gaussian noises are given. Then the LO detector test statistic is derived in section 3. In section 4 , based on the new acquisition scheme, the numerical results of detection probability are given.

\section{Observation Model}

Considering the multiplicative and weakly dependent non-Gaussian noises model for a DSSS system, the received signal can be expressed as

$$
r(t)=s(t)+s(t) n(t)+w(t)
$$

where $s(t)$ is the primary direct-path signal; $n(t)$ is the additional multiplicative noise which may be characterizing the fading amplitude characteristics of secondary propagation paths; $w(t)$ is the weakly dependent non-Gaussian noise, $n(t)$ is a random variables independent of $s(t)$ but generally correlated with $w(t)$. And $s(t)$ can be expressed as [1]

$$
s(t)=\sqrt{2 E} d\left(t-\tau T_{c}\right) c\left(t-\tau T_{c}\right) \cos \left(\omega_{c} t+\varphi\right)
$$

In equation (2), $E$ is the energy per chip; $d(t)$ is the data sequence waveform, and in this paper it is assumed that there is a preamble for acquisition so that no data modulation is present during acquisition (i.e., $d(t)=1) ; T_{c}$ is the chip duration; $\tau$ is the time delay

normalized by $T_{c} ; c(t)=\sum_{-\infty}^{\infty} c_{i} p_{T_{c}}\left(t-i T_{c}\right)$ where $c_{i} \in\{-1,+1\}$ is the $i$ th chip of a pseudonoise (PN) code sequence of period $L$ and $p_{T_{c}}(t)$ is the PN code waveform defined as a unit rectangular pulse over $\left[0, T_{c}\right] ; \omega_{c}$ is the carrier angular frequency; $\phi$ is the phase distributed uniformly over $[0,2 \pi)$.

A typical structure of the noncoherent I-Q correlators receiver is shown in Fig.1. We consider the serial search scheme with single dwell [1]. The $i$ th sampled I-Q components $X_{i}^{I}$ and $X_{i}^{Q}$ can be obtained as, for $i=1,2, \cdots, M$

$$
\begin{aligned}
& X_{i}^{I}=\int_{t_{i}-T_{c}}^{t_{c}} r(t) c\left(t-\hat{\tau} T_{c}\right) \sqrt{2} \cos \left(\omega_{c} t\right) d t \\
& X_{i}^{Q}=\int_{t_{i}-T_{c}}^{t_{t}} r(t) c\left(t-\hat{\tau} T_{c}\right) \sqrt{2} \sin \left(\omega_{c} t\right) d t
\end{aligned}
$$

respectively, where $M$ is the correlation length, $\hat{\tau}$ is the time delay (normalized by the chip duration $T_{c}$ ) of the locally generated PN code, and $t_{i}=t_{0}+i T_{c}$. Here, $t_{0}$ is an initial time. A test statistic is evaluated with the vectors $\left\{X_{i}^{I}\right\}_{i=1}^{M}$ and $\left\{X_{i}^{Q}\right\}_{i=1}^{M}$, and then compared with a threshold. In the conventional systems, the test statistic is, for example, the SS statistic $T_{S S}\left(X^{I}, X^{Q}\right)=\left(\sum_{i=1}^{M} X_{i}^{I}\right)^{2}+\left(\sum_{i=1}^{M} X_{i}^{Q}\right)^{2}$. 


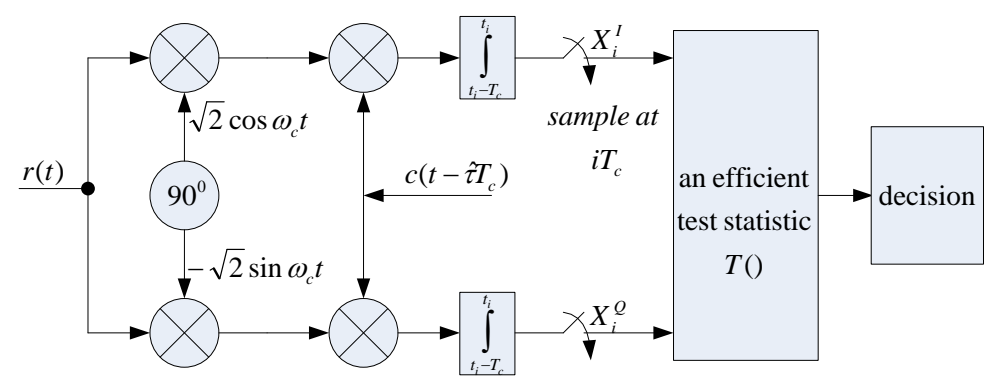

Figure 1. Structure of PN Code Acquisition System

The PN code acquisition problem can be regarded as a hypothesis testing problem: given $\left\{X_{i}^{I}\right\}_{i=1}^{M}$ and $\left\{X_{i}^{Q}\right\}_{i=1}^{M}$, a decision is to be made between the null hypothesis $H_{0}$ and alternative hypothesis $H_{1}$, where $H_{0}:|\tau-\hat{\tau}| \geq 1$ and $H_{1}:|\tau-\hat{\tau}|<1$. Under $H_{1}$, each sampled correlation value between the locally generated and received PN codes is $\sqrt{E}(1-|\delta|)$, where $\delta$ is the residual shift (normalized by $T_{c}$ ) between the two PN codes, with the value ranging in the interval $(-1,+1)$. For simplicity, we assume that the system is chip synchronous (that is, $\delta=0$ ) as in most other studies (an investigation of the effect of a nonzero $\delta$ has been considered in [15]). Thus, each sampled correlation value is $\sqrt{E}$. On the other hand, each sampled correlation value is +1 or -1 with equal probability, and the mean value of the sampled correlation is 0 under $H_{0}$. From these results and equation (3) and (4), $H_{0}$ and $H_{1}$ can be alternatively expressed as

$$
\begin{gathered}
H_{0}:\left(X_{i}^{I}=W_{i}^{I}, X_{i}^{Q}=W_{i}^{Q}\right), \quad i=1,2, \cdots M \\
H_{1}:\left(X_{i}^{I}=\theta \cos \varphi+\theta \cos \varphi N_{i}^{I}+W_{i}^{I}, X_{i}^{Q}=\theta \cos \varphi+\theta \cos \varphi N_{i}^{Q}+W_{i}^{Q}\right), \quad i=1,2, \cdots, M(6)
\end{gathered}
$$

Or simply described as

$$
\begin{aligned}
& H_{0}: \theta=0 \\
& H_{1}: \theta>0
\end{aligned}
$$

In equations (5)-(8), $\theta=\sqrt{E}$ is the signal strength parameter, $\left\{N_{i}^{b}\right\}_{i=1}^{M}$ and $\left\{W_{i}^{b}\right\}_{i=1}^{M}$ with $b=\{I, Q\}$ are the I-Q multiplicative noise and additive noise respectively. Let $f_{N^{I}}, f_{N^{Q}}$, $f_{W^{I}}$ and $f_{W^{Q}}$ be the common probability density function (PDF) of the I-Q multiplicative noise and additive noise, and $f_{N^{I} W^{I}}$ and $f_{N^{Q} W^{Q}}$ denote the common joint PDF of $\left(N^{I}, W^{I}\right)$ and $\left(N^{Q}, W^{Q}\right)$ respectively, which are i.i.d. random variables. Finally, $f_{\left(N^{I} W^{I}, N^{Q} W^{Q}\right)}$ and $f_{W}$ denote the joint PDF of observation noise of I-Q components under $H_{1}$, and $H_{0}$ respectively.

Assuming that the degree of dependence among noise components is weak, the dependentnoise components $\left\{W_{i}^{I}\right\}_{i=1}^{M}$ and $\left\{W_{i}^{Q}\right\}_{i=1}^{M}$ in this paper are modeled by the first-order moving average (FOMA) of i.i.d. random variables, which has been proved to be a simple and good approximation to the weakly dependent noise environment [16-17]. Specifically, let us assume that

$$
W_{i}^{I}=\Lambda_{i}^{I}+\rho \Lambda_{i-1}^{I}
$$




$$
W_{i}^{Q}=\Lambda_{i}^{Q}+\rho \Lambda_{i-1}^{Q}
$$

where $\rho$ is called the dependence parameter determining the correlation coefficient of $W_{i}^{I}$ and $W_{i}^{Q}$ especially, and $\left\{\Lambda_{i}^{I}\right\}_{i=1}^{M}$ and $\left\{\Lambda_{i}^{Q}\right\}_{i=1}^{M}$ are i.i.d. random process with $\Lambda_{0}^{I}=\Lambda_{0}^{Q}=0$. In this paper, We model $\Lambda^{I}$ and $\Lambda^{Q}$ with symmetric $\alpha$-stable ( $\mathrm{S} \alpha \mathrm{S}$ ) distributions, which have been proved to be very useful in modeling non-Gaussian noise [18]. The joint probability density function of $\Lambda_{i}{ }^{I}$ and $\Lambda_{i}{ }^{Q}$ can be most conveniently defined by the inverse Fourier transform (IFT) of their characteristic function [18]

$$
f_{\alpha, \gamma, \beta_{1}, \beta_{2}}\left(x_{1}, x_{2}\right)=\frac{1}{(2 \pi)^{2}} \int_{-\infty}^{\infty} \int_{-\infty}^{\infty} \exp \left[i\left(\beta_{1} \omega_{1}+\beta_{2} \omega_{2}\right)-\gamma\left(\omega_{1}{ }^{2}+\omega_{2}{ }^{2}\right)^{\frac{\alpha}{2}}\right] \mathrm{e}^{-i\left(x_{1} \omega_{1}+x_{2} \omega_{2}\right)} d \omega_{1} d \omega_{2}
$$

where the parameter $\alpha$ and $\gamma$ are termed the characteristic exponent and dispersion, respectively, and $\beta_{1}$ and $\beta_{2}$ are location parameters. The characteristic exponent $\alpha$ ranges in the interval $0<\alpha \leq 2$ with a smaller $\alpha$ indication heavier tails (more impulsive behavior). The dispersion $\gamma$ is a positive constant relation to the spread of the PDF. The location parameters $\beta_{1}$ and $\beta_{2}$ are the symmetry axes of the marginal $\mathbf{S} \alpha \mathbf{S}$ distributions: we assume $\beta_{1}=\beta_{2}=0$, without loss of generality. Unfortunately, no closed-form expression exists for (11), except for the special cases of $\alpha=1$ and $\alpha=2$

$$
f_{\alpha, \gamma}\left(x_{1}, x_{2}\right)= \begin{cases}\frac{\gamma}{2 \pi\left(x_{1}^{2}+x_{2}^{2}+\gamma^{2}\right)^{\frac{3}{2}}} & \text { for } \alpha=1 \\ \frac{1}{4 \pi \gamma} \exp \left(-\frac{x_{1}^{2}+x_{2}^{2}}{4 \gamma}\right) & \text { for } \alpha=2\end{cases}
$$

Because of such a lack of closed-form expressions, we concentrate on the case $\alpha=1$. Nonetheless, we shall see in Section 4 that the system obtained for $\alpha=1$ is not only robust to the variation of $\alpha$, but also outperforms the conventional system for most values of $\alpha$.

Assuming that the samples $\left\{X_{i}^{I}, X_{i}^{Q}\right\}$ of the bivariate noise process form a sequence of independent random vectors for a given $\phi$, these joint PDF of $2 M$ sampled in-phase and quadrature observations $\left\{X_{i}^{I}, X_{i}^{Q}\right\}, i=1,2, \cdots, M$ is

$$
\begin{aligned}
f_{X^{I}, X^{Q}}\left(X^{I}, X^{Q}\right) & =E_{\phi}\left\{\int f_{\left(N^{I} W^{I}, N^{Q} W^{Q}\right)} d N\right\} \\
& =E_{\phi}\left\{\prod_{i=1}^{M} \iint f_{\left(n_{i}^{I}, y_{i}^{I}(\theta)\right),\left(n_{i}^{Q}, y_{i}^{Q}(\theta)\right)} d n_{i}^{I} d n_{i}^{Q}\right\}
\end{aligned}
$$

Where $E_{\phi}$ denotes the expectation over $\phi$, and $y_{i}^{I}$ and $y_{i}^{Q}$ can be expressed as

$$
\begin{aligned}
& y_{i}^{I}(\theta)=x_{i}^{I}-\theta \cos \phi-n_{i}^{I} \theta \cos \phi \\
& y_{i}^{Q}(\theta)=x_{i}^{Q}-\theta \cos \phi-n_{i}^{Q} \theta \cos \phi
\end{aligned}
$$

Substituting equations (9) and (10) into equation (13), the joint PDF of $2 M$ sampled inphase and quadrature observations $\left\{X_{i}^{I}, X_{i}^{Q}\right\}$ can be rewritten as follow 


$$
\begin{aligned}
f_{X^{I}, X^{Q}}\left(x^{I}, x^{Q}\right)= & E_{\phi}\left\{\prod_{i=1}^{M} \iint f_{\left(n_{i}^{I}, y_{i}^{I}(\theta)\right),\left(n_{i}^{Q}, y_{i}^{Q}(\theta)\right)} d n_{i}^{I} d n_{i}^{Q}\right\} \\
= & E_{\phi}\left\{\iint f_{\left(n_{1}^{I}, y_{1}^{I}(\theta)\right),\left(n_{1}^{Q}, y_{1}^{Q}(\theta)\right)} d n_{1}^{I} d n_{1}^{Q} \times \iint f_{\left(n_{2}^{I}, y_{2}^{I}(\theta)-\rho y_{1}^{I}(\theta)\right),\left(n_{2}^{Q}, y_{2}^{Q}(\theta)-\rho y_{1}^{Q}(\theta)\right)} d n_{2}^{I} d n_{2}^{Q}\right. \\
& \times \iint f_{\left(n_{3}^{I}, y_{3}^{I}(\theta)-\rho y_{2}^{I}(\theta)+\rho^{2} y_{1}^{I}(\theta)\right),\left(n_{3}^{Q}, y_{3}^{Q}(\theta)-\rho y_{2}^{Q}(\theta)+\rho^{2} y_{1}^{Q}(\theta)\right)} d n_{3}^{I} d n_{3}^{Q} \times \cdots \times \\
& \left.\iint f_{\left(n_{M}^{I}, y_{M}^{I}(\theta)-\rho y_{M-1}^{I}(\theta)+\cdots+(-\rho)^{M-1} y_{1}^{I}(\theta)\right),\left(n_{M}^{Q}, y_{M}^{Q}(\theta)-\rho y_{M-1}^{Q}(\theta)+\cdots+(-\rho)^{M-1} y_{1}^{Q}(\theta)\right)} d n_{M}^{I} d n_{M}^{Q}\right\} \\
= & E_{\phi}\left\{\prod_{i=1}^{M} \iint f_{\left(n_{i}^{I}, Y_{i}^{I}-C_{i} \theta \cos \phi-U_{i}^{I} \theta \cos \phi\right),\left(n_{i}^{Q}, Y_{i}^{Q}-C_{i} \theta \sin \phi-U_{i}^{Q} \theta \sin \phi\right)} d n_{i}^{I} d n_{i}^{Q}\right\}
\end{aligned}
$$

where $C_{i}=\sum_{j=0}^{i-1}(-\rho)^{j}, U_{i}^{I}=-\sum_{j=0}^{i-1}(-\rho)^{j} n_{i-j}^{b}$ and $Y_{i}^{b}=\sum_{j=0}^{i-1}(-\rho)^{j} X_{i-j}^{b}$ for $b=\{I, Q\}$. Then the null hypothesis $H_{0}$ and alternative hypothesis $H_{1}$ can be transformed as for $i=1,2, \cdots M$

$$
\begin{aligned}
& H_{0}:\left(Y_{i}^{I}=\Lambda_{i}^{I}, Y_{i}^{Q}=\Lambda_{i}^{Q}\right) \\
& H_{1}:\left(Y_{i}^{I}=\theta \cos \varphi C_{i}+U_{i}^{I} \theta \cos \varphi+\Lambda_{i}^{I}, Y_{i}^{Q}=\theta \sin \varphi C_{i}+U_{i}^{Q} \theta \sin \varphi+\Lambda_{i}^{Q}\right)
\end{aligned}
$$

And the joint PDF of $2 M$ sampled in-phase and quadrature observations $\left\{Y_{i}^{I}, Y_{i}^{Q}\right\}_{i=1}^{M}$ is

$$
\begin{aligned}
f_{Y^{I}, Y^{Q}}\left(Y^{I}, Y^{Q}\right) & =E_{\varphi}\left\{\int f_{\left(U^{I} \Lambda^{l}, U^{Q} \Lambda^{Q}\right)} d U\right\} \\
& =E_{\varphi}\left\{\prod_{i=1}^{M} \iiint_{\left(U_{i}^{l}, V_{i}^{I}(\theta)\right),\left(U_{i}^{Q}, V_{i}^{Q}(\theta)\right)} d U_{i}^{I} d U_{i}^{Q}\right\}
\end{aligned}
$$

Where

$$
\begin{aligned}
& V_{i}^{I}(\theta)=Y_{i}^{I}-\theta \cos \varphi C_{i}-U_{i}^{I} \theta \cos \varphi \\
& V_{i}^{Q}(\theta)=Y_{i}^{Q}-\theta \sin \varphi C_{i}-U_{i}^{Q} \theta \operatorname{Sin} \varphi
\end{aligned}
$$

\section{LO Detector Test Statistic}

The test statistic of an LO detector is obtained as $[6,12]$

$$
T_{L O}\left(Y^{I}, Y^{Q}\right)=\frac{1}{\left.f_{Y^{I}, Y^{Q}}\left(Y^{I}, Y^{Q}\right)\right|_{\theta=0}} \times\left.\frac{d^{v} f_{Y^{I}, Y^{Q}}\left(Y^{I}, Y^{Q}\right)}{d \theta^{v}}\right|_{\theta=0}
$$

where $v$ is the order of the first nonzero derivative of $f_{Y^{I}, Y^{Q}}\left(Y^{I}, Y^{Q}\right)$ at $\theta=0$. As shown in the appendix, the test statistic of the LO detector is

$$
\begin{aligned}
T_{P_{-} L O}\left(X^{I}, X^{Q}\right) & =\left.T_{L O}\left(Y^{I}, Y^{Q}\right)\right|_{Y_{i}^{b}=\sum_{j=0}^{L-1}(-\rho)^{j} X_{i-j}^{b}} \\
& =\sum_{i=1}^{M}\left\{h\left(Y_{i}^{I}\right)+h\left(Y_{i}^{Q}\right)\right\}+\sum_{i=1}^{M} \sum_{j \neq i, j=1}^{M}\left\{g\left(Y_{i}^{I}\right) g\left(Y_{j}^{I}\right)+g\left(Y_{i}^{Q}\right) g\left(Y_{j}^{Q}\right)\right\}
\end{aligned}
$$

where

$$
\begin{array}{r}
h\left(Y_{i}^{b}\right)=\frac{1}{f_{\Lambda}\left(Y_{i}^{I}, Y_{i}^{Q}\right)} \times \frac{\partial^{2}\left(f_{\Lambda}\left(Y_{i}^{I}, Y_{i}^{Q}\right) E\left\{\left(C_{i}+U^{b}\right)^{2} \mid \Lambda=Y_{i}^{I}, Y_{i}^{Q}\right\}\right)}{\partial\left(Y_{i}^{b}\right)^{2}} \\
g\left(Y_{i}^{b}\right)=\frac{1}{f_{\Lambda}\left(Y_{i}^{I}, Y_{i}^{Q}\right)} \times \frac{\partial\left(f_{\Lambda}\left(Y_{i}^{I}, Y_{i}^{Q}\right) E\left\{\left(C_{i}+U^{b}\right) \mid \Lambda=Y_{i}^{I}, Y_{i}^{Q}\right\}\right)}{\partial Y_{i}^{b}}
\end{array}
$$


for $b=\{I, Q\}$.

Compared with the test statistic of the LO detector in the additive and independent noise environments [13], it is noteworthy that the multiplicative noise introduces the extra terms $E\left\{\left(C_{i}+U^{b}\right)^{2} \mid \Lambda=Y_{i}^{I}, Y_{i}^{Q}\right\}$ and $\left.E\left\{\left(C_{i}+U^{b}\right) \mid \Lambda=Y_{i}^{I}, Y_{i}^{Q}\right\}\right)$ for each $h\left(Y_{i}^{b}\right)$ and $g\left(Y_{i}^{b}\right)$ in the test statistic, and the dependent noises introduces the new variables $Y_{i}^{b}, U_{i}^{b}$ and $C_{i}$. If there is no multiplicative noise $(N=0)$ and the noise is independent, the proposed LO detector has the same test statistic with the LO detector work in non-Gaussian channels which researched in [13]. Since the FOMA noise model is a simple and good approximation to the weakly dependent noise environment, we can ignore the high-order terms of $\rho\left(\left\{\rho^{i} \mid i=2,3 \cdots M\right\}\right)$ to get the simpler-LO detector. And then the test statistic of simpler-LO detector can be obtained

$$
T_{S L O}\left(X^{I}, X^{Q}\right)=\left.T_{L O}\left(Y^{I}, Y^{Q}\right)\right|_{Y_{i}^{b}=X_{i}^{b}-\rho X_{i-1}^{b}, C_{i}=1-\rho u(i-2)} i=(1,2 \cdots M)
$$

In equation (26) $X_{0}^{b}=0$ and $b=\{I, Q\}$, and $u(i)$ is the unit step sequence, i.e., $u(i)=0$ when $i<0$ and $u(i)=1$ when $i \geq 0$.

\section{Simulation Results}

In this section, the performance of the proposed simpler-LO (PS-LO) detector and conventional SS detector and the Non-Gaussian Additive and Independent LO (NAI-LO) detector [13] is simulated with a PN code of $L=1023$ chips, generated form an $m$ sequence with the primitive polynomial $1+z^{3}+z^{10}$ when $M=50$. Because it is difficult to get explicit closed-form formulations for finite sample-size performance, we resorted to Monte Carlo simulations to show the results.

In our simulations, we will consider a specific example to illustrate the results, we assume $N^{b}=r s W^{b}$ for $b=\{I, Q\} . r$ is the correlation coefficient between $N^{b}$ and $W^{b}$, and $N^{I}, N^{Q}$ have same variance $\sigma_{N^{I}}^{2}=\sigma_{N^{Q}}^{2}=s^{2}$. Noise samples $\left\{\Lambda_{i}^{I}\right\}$ and $\left\{\Lambda_{i}^{Q}\right\}$ are generated from [19]

$$
\gamma^{\frac{1}{\alpha}} \frac{\sin (\alpha A)}{(\cos A)^{\frac{1}{\alpha}}}\left(\frac{\cos [(1-\alpha) A]}{B}\right)^{\frac{1-\alpha}{\alpha}}
$$

where $A$ is uniform on $(-\pi / 2, \pi / 2)$ and $B$ is exponential with mean 1. Since $\mathbf{S} \alpha \mathbf{S}$ noise with $\alpha<2$ has no finite variance, making the standard SNR measure inconsistent, a new scale parameter is used to indicate the strength of the $\mathbf{S} \alpha \mathbf{S}$ noise. The new SNR, which provides a mathematically and conceptually valid characterization of the relative strength between the information-bearing signal and channel noise with infinite variance, is defined as

$$
\frac{1}{2 C_{g}\left(1+\rho^{2}\right)}\left(\frac{\sqrt{E}}{S_{0}}\right)^{2}
$$

where $S_{0}=\left(C_{g} \gamma\right)^{1 / \alpha} / C_{g}$ with $C_{g} \approx 1.78$ the exponential of the Euler's constant $\left(\lim _{n \rightarrow \infty}\left(\sum_{k=1}^{n}(1 / k)-\ln n\right)\right)$. The normalizing constant $2 C_{g}\left(1+\rho^{2}\right)$ ensures that the definition of the SNR coincides with that of the standard SNR in the independent Gaussian case. Because $\gamma$ can be easily and exactly estimated using only the sample mean and variance of 
observations $X^{I}$ or $X^{Q}$ [20], it may be regarded as a known value: in our simulations, we assume $\gamma=1$.

Each point in Figure 2, 3, 4and 5 was obtained from 100000 runs to make the relative error less than $1 \%$ for a false-alarm probability $P_{f a}=10^{-2}$. Figure 2 shows the detection probabilities of conventional SS detector, the NAI-LO detector and the proposed Simpler-LO detector for some values of $r s$ and dependent parameter $\rho$ in multiplicative and weakly dependent non-Gaussian noises for $\alpha=1$. Figure 3 shows the detection probabilities plotted as a function of $\rho$ for some values of $\mathrm{SNR} /$ chip and $r s$ in multiplicative and weakly dependent non-Gaussian noises for $\alpha=1$. Figure 4 shows the detection probabilities plotted as a function of $r s$ for some values of $\mathrm{SNR} /$ chip and $\rho$ in multiplicative and weakly dependent non-Gaussian noises for $\alpha=1$. From these plots, it is observed that the proposed detector significantly outperforms the conventional SS detector and the NAI-LO detector in multiplicative and weakly dependent non-Gaussian noises.

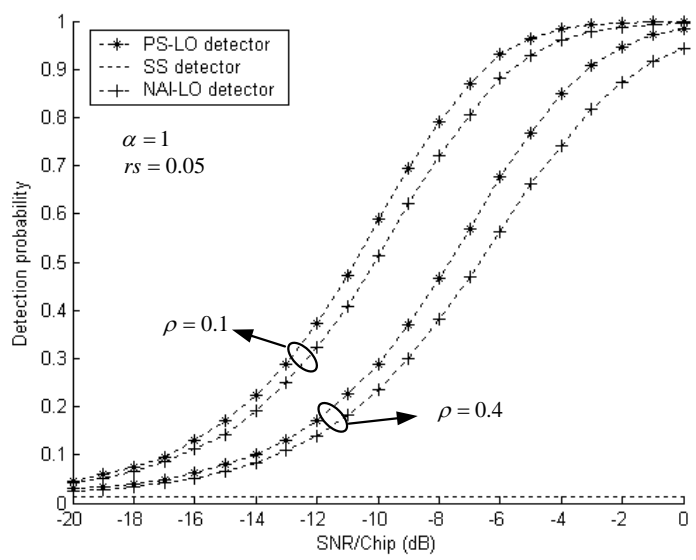

Figure 2. Detection Probabilities of the Conventional SS Detector, the NAI-LO Detector and the Proposed Schemes for Different $r s$ and $\rho$ when $P_{f a}=10^{-2}$ and $M=50$ in Multiplicative and Weakly Dependent Non-Gaussian Noises

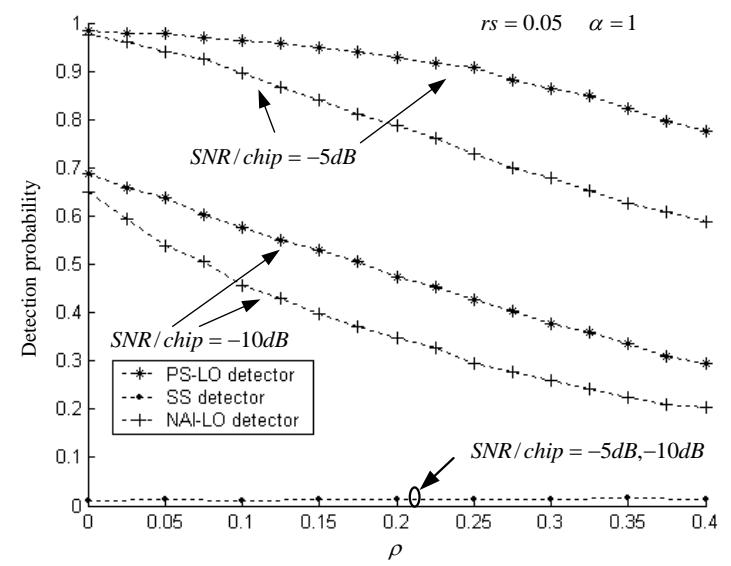

Figure 3. Detection Probabilities Plotted as a Function of $\rho$ for some Values of SNR/chip and $r s$ when $P_{f a}=10^{-2}$ and $M=50$ in Multiplicative and Weakly

Dependent Non-Gaussian Noises for $\alpha=1$ 
Figure 5 shows the detection probabilities of the detectors as a function of $\alpha$ for some values of $\rho, r s$ and $\mathrm{SNR} /$ chip. We can clearly see that the proposed simpler-LO detector significantly outperforms the conventional SS detector for most values of $\alpha$, and performs better than the NAI-LO detector for all values of $\alpha$. Only when $\alpha$ is close to two, the conventional SS detector performs slightly better. Another important observation is that the performance of proposed simpler-LO detector are robust to the variation of the value of $\alpha$. In addition, the proposed detector performs better as the impulsiveness becomes higher. This can be explained as follows, Impulses which have large amplitudes are clipped in the proposed detector. Therefore, the effective noise variance at the output of the proposed detector is smaller than the total input noise variance.

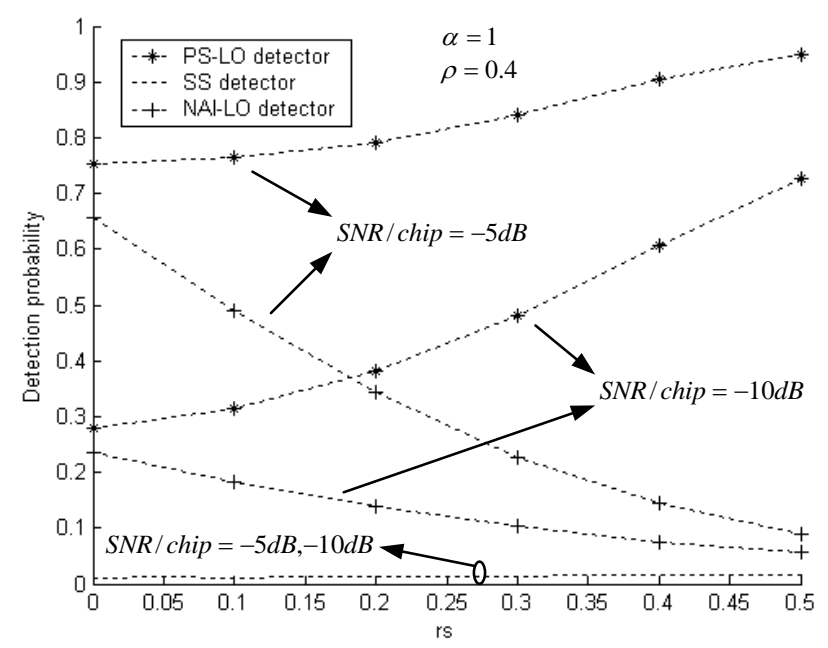

Figure 4. Detection Probabilities Plotted as a Function of $r s$ for Some Values of SNR/chip and $\rho$ when $P_{f a}=10^{-2}$ and $M=50$ in Multiplicative and Weakly Dependent Non-Gaussian Noises for $\alpha=1$

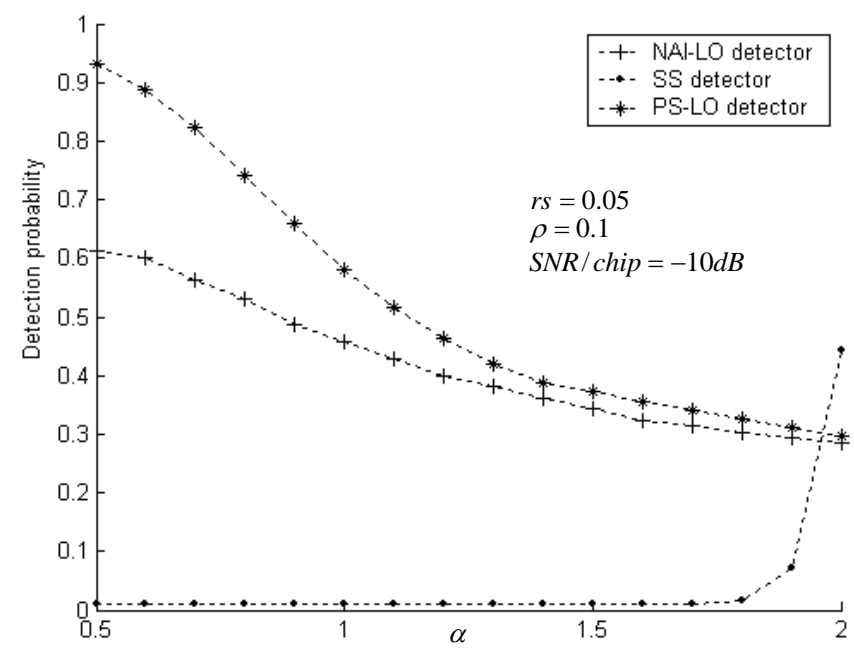

Figure 5. Detection Probabilities of the Detectors as a Function of $\alpha$ for Some Values of $\rho, r s$ and SNR/chip when $P_{f a}=10^{-2}$ and $M=50$ 


\section{Conclusions}

In this paper, a new detector for DS-SS code acquisition in the environments with multiplicative and weakly dependent non-Gaussian noises has been proposed. Using the locally optimum test statistic, a decision rule of code acquisition has been derived and a new acquisition system has been proposed based on the decision rule. The performance of the proposed scheme has been compared with that of the conventional schemes. From the simulation results, it has been observed that the proposed scheme significantly outperforms the conventional SS detector and the NAI-LO detector in multiplicative and weakly dependent non-Gaussian noises.

\section{Appendix}

Derivation of the LO detector test statistic We have defined

so we can get

$$
\begin{gathered}
V_{i}^{I}(\theta)=Y_{i}^{I}-\theta \cos \varphi C_{i}-U_{i}^{I} \theta \cos \varphi \\
V_{i}^{Q}(\theta)=Y_{i}^{Q}-\theta \sin \varphi C_{i}-U_{i}^{Q} \theta \operatorname{Sin} \varphi
\end{gathered}
$$

$$
\left.V_{i}^{b}(\theta)\right|_{\theta=0}=V_{i}^{b}(0)=Y_{i}^{b}
$$

for $b=\{I, Q\}$.

We also define

$$
\begin{gathered}
f_{i}(\theta)=f_{\left(U_{i}^{I}, V_{i}^{I}(\theta)\right),\left(U_{i}^{Q}, V_{i}^{Q}(\theta)\right)} \\
f_{i}(0)=f_{\left(U_{i}^{I}, V_{i}^{I}(0)\right),\left(U_{i}^{Q}, V_{i}^{Q}(0)\right)} \\
f_{i}^{\prime}(0)=f_{\left(U_{i}^{I}, V_{i}^{I}(0)\right),\left(U_{i}^{Q}, V_{i}^{Q}(0)\right)}^{\prime}
\end{gathered}
$$

and $f_{i}^{\prime \prime}(0)=f_{\left(U_{i}^{I}, V_{i}^{I}(0)\right),\left(U_{i}^{Q}, V_{i}^{Q}(0)\right)}^{\prime \prime}$.

The first derivative of $f_{Y^{I}, Y^{Q}}\left(Y^{I}, Y^{Q}\right)$ at $\theta=0$ is

$$
\left.\frac{d f_{Y^{I}, Y^{Q}}\left(Y^{I}, Y^{Q}\right)}{d \theta}\right|_{\theta=0}=E_{\varphi}\left\{\sum_{i=1}^{M} \iint f_{i}^{\prime}(0) d U_{i}^{I} d U_{i}^{Q} \prod_{j \neq i, j=1}^{M} \iint f_{j}(0) d U_{j}^{I} d U_{j}^{Q}\right\}
$$

where

$$
\frac{d f_{i}(\theta)}{d \theta}=\frac{\partial f_{i}(\theta)}{\partial V_{i}^{I}(\theta)} \times \frac{d V_{i}^{I}(\theta)}{d \theta}+\frac{\partial f_{i}(\theta)}{\partial V_{i}^{Q}(\theta)} \times \frac{d V_{i}^{Q}(\theta)}{d \theta}
$$

where

$$
\begin{aligned}
& \frac{d V_{i}^{I}(\theta)}{d \theta}=\frac{d\left(Y_{i}^{I}-C_{i} \theta \cos \varphi-U_{i}^{I} \theta \cos \varphi\right)}{d \theta}=-C_{i} \cos \varphi-U_{i}^{I} \cos \varphi \\
& \frac{d V_{i}^{Q}(\theta)}{d \theta}=\frac{d\left(Y_{i}^{Q}-C_{i} \theta \sin \varphi-U_{i}^{Q} \theta \sin \varphi\right)}{d \theta}=-C_{i} \sin \varphi-U_{i}^{Q} \sin \varphi
\end{aligned}
$$

So

and

$$
\begin{aligned}
\left.\frac{d f_{i}(\theta)}{d \theta}\right|_{\theta=0} & =\left.\left\{-\left(C_{i}+U_{i}^{I}\right) \cos \phi \times \frac{\partial f_{i}(\theta)}{\partial V_{i}^{I}(\theta)}-\left(C_{i}+U_{i}^{Q}\right) \sin \phi \times \frac{\partial f_{i}(\theta)}{\partial V_{i}^{Q}(\theta)}\right\}\right|_{\theta=0} \\
& =-\left(C_{i}+U_{i}^{I}\right) \cos \phi \times \frac{\partial f_{i}(0)}{\partial Y_{i}^{I}}-\left(C_{i}+U_{i}^{Q}\right) \sin \phi \times \frac{\partial f_{i}(0)}{\partial Y_{i}^{Q}}
\end{aligned}
$$

$$
\begin{aligned}
E_{\phi}\left\{f_{i}^{\prime}(\theta)\right\} & =E_{\phi}\left\{-\left(C_{i}+U_{i}^{I}\right) \cos \phi \times \frac{\partial f_{i}(0)}{\partial Y_{i}^{I}}-\left(C_{i}+U_{i}^{Q}\right) \sin \phi \times \frac{\partial f_{i}(0)}{\partial Y_{i}^{Q}}\right\} \\
& =0
\end{aligned}
$$


So the first derivative of $f_{Y^{I}, Y^{Q}}\left(Y^{I}, Y^{Q}\right)$ at $\theta=0$ is vanishes. We thus obtain the second derivative of $f_{Y^{I}, Y^{Q}}\left(Y^{I}, Y^{Q}\right)$ at $\theta=0$

where

$$
\begin{aligned}
& \left.\frac{d^{2} f_{Y^{I}, Y^{Q}}\left(Y^{I}, Y^{Q}\right)}{d \theta^{2}}\right|_{\theta=0}=E_{\phi}\left\{\sum _ { i = 1 } ^ { M } \left[\iint f_{i}^{\prime \prime}(0) d U_{i}^{I} d U_{i}^{Q} \prod_{j \neq i, j=1}^{M} \iint f_{j}(0) d U_{j}^{I} d U_{j}^{Q}\right.\right. \\
& \left.\left.+\iint f_{i}^{\prime}(0) d U_{i}^{I} d U_{i}^{Q} \sum_{j=1, j \neq i}^{M} \iint f_{j}^{\prime}(0) d U_{j}^{I} d U_{j}^{Q} \prod_{k=1, k \neq i, j}^{M} \iint_{k} f_{k}(0) d U_{k}^{I} d U_{k}^{Q}\right]\right\}
\end{aligned}
$$

$$
\begin{aligned}
& \frac{d^{2} f_{i}(\theta)}{d \theta^{2}}=\left(C_{i}+U_{i}^{I}\right)^{2} \cos ^{2} \phi \times \frac{\partial^{2} f_{i}(\theta)}{\partial\left(V_{i}^{I}(\theta)\right)^{2}}+\left(C_{i}+U_{i}^{Q}\right)^{2} \sin ^{2} \phi \frac{\partial^{2} f_{i}(\theta)}{\partial\left(V_{i}^{Q}(\theta)\right)^{2}}+ \\
& \left(C_{i}+U_{i}^{I}\right)\left(C_{i}+U_{i}^{Q}\right) \sin \phi \cos \phi \frac{\partial^{2} f_{i}(\theta)}{\partial\left(V_{i}^{I}(\theta)\right) \partial\left(V_{i}^{Q}(\theta)\right)}+\left(C_{i}+U_{i}^{I}\right)\left(C_{i}+U_{i}^{Q}\right) \sin \phi \cos \phi \frac{\partial^{2} f_{i}(\theta)}{\partial\left(V_{i}^{Q}(\theta)\right) \partial\left(V_{i}^{I}(\theta)\right)}
\end{aligned}
$$

the first term of (38) is

$$
E_{\varphi}\left\{\sum_{i=1}^{M} \iint f_{i}^{\prime \prime}(0) d U_{i}^{I} d U_{i}^{Q} \prod_{j \neq i, j=1}^{M} \iint f_{j}(0) d U_{j}^{I} d U_{j}^{Q}\right\}=\frac{1}{2} \sum_{i=1}^{M}\left\{h\left(Y_{i}^{I}\right)+h\left(Y_{i}^{Q}\right)\right\} \prod_{j=1}^{M} \iint f_{j}(0) d U_{j}^{I} d U_{j}^{Q}
$$

where

$$
\begin{aligned}
h\left(Y_{i}^{b}\right) & =\left.\left\{\iint\left(C_{i}+U_{i}^{b}\right)^{2} \frac{\partial^{2} f_{i}(\theta)}{\partial\left(V_{i}^{b}(\theta)\right)^{2}} \times \frac{1}{\iint f_{i}(\theta) d U_{i}^{I} d U_{i}^{Q}} d U_{i}^{I} d U_{i}^{Q}\right\}\right|_{\theta=0} \\
& =\left.\left\{\frac{1}{\iint f_{i}(\theta) d U_{i}^{I} d U_{i}^{Q}} \times \frac{\partial^{2}\left(\iint\left(C_{i}+U_{i}^{b}\right)^{2} f_{i}(\theta) d U_{i}^{I} d U_{i}^{Q}\right)}{\partial\left(V_{i}^{b}(\theta)\right)^{2}}\right\}\right|_{\theta=0} \\
& =\frac{1}{f_{\Lambda}\left(Y^{I}, Y^{Q}\right)} \times \frac{\partial^{2}\left(f_{\Lambda}\left(Y^{I}, Y^{Q}\right) E\left\{\left(C_{i}+U^{b}\right)^{2} \mid \Lambda=Y^{I}, Y^{Q}\right\}\right)}{\partial\left(Y^{b}\right)^{2}}
\end{aligned}
$$

for $b=\{I, Q\}$.

Similarly, noting that

$$
\begin{aligned}
f_{i}^{\prime}(\theta) f_{j}^{\prime}(\theta)= & \cos ^{2} \varphi\left(C_{i}+U_{i}^{I}\right)\left(C_{j}+U_{j}^{I}\right) \frac{\partial f_{i}(\theta) \partial f_{j}(\theta)}{\partial V_{i}^{I}(\theta) \partial V_{j}^{I}(\theta)}+\sin ^{2} \varphi\left(C_{i}+U_{i}^{Q}\right)\left(C_{j}+U_{j}^{Q}\right) \frac{\partial f_{i}(\theta) \partial f_{j}(\theta)}{\partial V_{i}^{Q}(\theta) \partial V_{j}^{Q}(\theta)} \\
& +\sin \varphi \cos \varphi\left(C_{i}+U_{i}^{I}\right)\left(C_{j}+U_{j}^{Q}\right)\left(\frac{\partial f_{i}(\theta) \partial f_{j}(\theta)}{\partial V_{i}^{I}(\theta) \partial V_{j}^{Q}(\theta)}+\frac{\partial f_{i}(\theta) \partial f_{j}(\theta)}{\partial V_{i}^{Q}(\theta) \partial V_{j}^{I}(\theta)}\right)
\end{aligned}
$$

the second term of (38) is

$$
\begin{aligned}
& E_{\varphi}\left\{\sum_{i=1}^{M} \sum_{j=1, j \neq i}^{M} \iint f_{i}^{\prime}(0) d U_{i}^{I} d U_{i}^{Q} \iint f_{j}^{\prime}(0) d U_{j}^{I} d U_{j}^{Q} \prod_{k=1, k \neq i, j}^{M} \iint f_{k}(0) d U_{k}^{I} d U_{k}^{Q}\right\} \\
& =\frac{1}{2} \sum_{i=1}^{M} \sum_{j=1, j \neq i}^{M}\left\{g\left(Y_{i}^{I}\right) g\left(Y_{j}^{I}\right)+g\left(Y_{i}^{Q}\right) g\left(Y_{j}^{Q}\right)\right\} \times \prod_{k=1}^{M} \iint f_{k}(0) d U_{k}^{I} d U_{k}^{Q}
\end{aligned}
$$

where

$$
\begin{aligned}
& g\left(Y_{i}^{b}\right)=\left.\left\{\iint\left(C_{i}+U_{i}^{b}\right) \frac{\partial f_{i}(\theta)}{\partial V_{i}^{b}(\theta)} \times \frac{1}{\iint f_{i}(\theta) d U_{i}^{I} d U_{i}^{Q}} d U_{i}^{I} d U_{i}^{Q}\right\}\right|_{\theta=0}= \\
& \left.\left\{\frac{1}{\iint f_{i}(\theta) d U_{i}^{I} d U_{i}^{Q}} \times \frac{\partial\left(\iint\left(1+U_{i}^{b}\right) f_{i}(\theta) d U_{i}^{I} d U_{i}^{Q}\right)}{\partial V_{i}^{b}(\theta)}\right\}\right|_{\theta=0} \\
& =\frac{1}{f_{\Lambda}\left(Y^{I}, Y^{Q}\right)} \times \frac{\partial\left(f_{\Lambda}\left(Y^{I}, Y^{Q}\right) E\left\{\left(C_{i}+U^{b}\right) \mid \Lambda=Y^{I}, Y^{Q}\right\}\right)}{\partial Y^{b}}
\end{aligned}
$$

for $b=\{I, Q\}$. 


Using (40) and (43) in (38), and then dividing the result by
$\left.f_{Y^{I}, Y^{Q}}\left(Y^{I}, Y^{Q}\right)\right|_{\theta=0}=\prod_{k=1}^{M} \iint f_{k}(0) d U_{k}^{I} d U_{k}^{Q}$, we can get $(23)$.

\section{Acknowledgements}

This work was supported by National Natural Science Foundation of China (Grant No. 61102107, 61374208), the Fundamental Research Funds for the Central Universities (HEUCFX41310).

\section{References}

[1] R. R. Rick and L. B. Milstein, "Parallel acquisition in mobile DS-CDMA systems", IEEE Trans. Commun.. vol. 45, no. 11, (1997).

[2] K. Choi, K. Cheun, and T. Jung, "Adaptive PN code acquisition using instantaneous power-scaled detection threshold under Rayleigh fading and pulsed Gaussian noise jamming”, IEEE Trans. Commun.. vol. 50, no. 8, (2002).

[3] P. Delaney, "Signal detection in multivariate class-A interference", IEEE Transactions on Communications, vol. 43, 2/3/4(1995).

[4] I. Song and S. A. Kassam, "Locally optimal detection of signals in a Generalized observation model: the known signal case”, IEEE Trans. Inf. Theory, vol. 36, no. 3, (1990).

[5] I. Song and S. A. Kassam, "Locally optimal detection of signals in a Generalized observation model: the random signal case", IEEE Trans. Inf. Theory, vol. 36, no. 3, (1990).

[6] I. Song, J. Bae, and S. Y. Kim, "Advanced Theory of Signal Detection”, Berlin, Springer-Verlag, Germany (2002).

[7] J. Lee, I. Song, H. Kwon, and H. J. Kim, "Locally Optimum Detection of Signal in Multiplicative and Firstorder Markov Additive Noises", IEEE Trans. Inf. Theory, vol. 54, no. 1, (2008).

[8] I. J. Kim, S. R. Park and I. Song, "Detection Schemes for Weak Signals in First-Order Moving Average of Impulsive Noise", IEEE Trans. Vehicular Technology, vol. 56, no. 1, (2007).

[9] J. Capon, "On the asymptotic efficiency of locally optimum detectors", IRE Trans. Inform. Theory. IT-7, vol. 4, (1961).

[10] H. V. Poor, “An Introduction to Signal Detection and Estimation”, Springer-Verlag, New York, (1988).

[11] S. A. Kassam, "Signal Detection in Non-Gaussian Noise", Springer-Verlag, New York (1988).

[12] W. E. Jacklin and D. R. Ucci, "Locally optimum detection and its application to communications and signal processing", H. Stark and P. M. Clarkson, Eds. Academic Press, San Diego (1995).

[13] S. Yoon, I. Song, and S. Y. Kim, "Code Acquisition for DS/SS Communications in Non-Gaussian Impulsive Channels", IEEE Trans. Commun., vol. 52, no. 2, (2004).

[14] A. B. Martinez, P. F. Swaszek, and J. B. Thomas, "Locally optimal detection in multivariate non-Gaussian noise", IEEE Trans. Inf. Theory, IT-30, no. 6, (1984).

[15] S. Yoon, I. Song, S. Y. Kim, and S. R. Park, "A DS/CDMA code-acquisition scheme robust to residual code phase offset variation”, IEEE Trans. Veh. Technol., vol. 49, no. 11, (2000).

[16] G. V. Moustakides and J. B. Thomas, "Min-max detection of weak signals in $\varphi$-mixing noise", IEEE Trans. Inf. Theory, IT-30, no. 3, (1984).

[17] H. V. Poor and J. B. Thomas, "Advances in Statistical Signal Processing Signal Detection”, vol. 2, Greenwich, CT: JAI, (1993).

[18] G. Samorodnitsky and M. S. Taqqu, "Stable Non-Gaussian Random Processes: Stochastic Models with Infinite Variance", Chapman \& Hall, New York (1994).

[19] A. D. Spaulding, "Locally optimum and suboptimum detector performance in a non-Gaussian interference environment", IEEE Trans. Commun., vol. 33, no. 4, (1985).

[20] X. Ma and C. L. Nikias, "Parameter estimation and blind channel identification in impulsive signal environments", IEEE Trans. Signal Processing, vol. 43, no. 12, (1995). 
International Journal of Hybrid Information Technology Vol.8, No.4 (2015) 